\title{
Sight-reading of violinists: eye movements anticipate the musical flow
}

\author{
Pascal Wurtz · René M. Mueri • Mario Wiesendanger
}

\begin{abstract}
When sight-reading a piece of music the eyes constantly scan the score slightly ahead of music execution. This separation between reading and acting is commonly termed eye-hand span and can be expressed in two ways: as anticipation in notes or in time. Previous research, predominantly in piano players, found skill-dependent differences of eye-hand span. To date no study has explored visual anticipation in violinists. The present study investigated how structural properties of a piece of music affect the eyehand span in a group of violinists. To this end eye movements and bow reversals were recorded synchronously while musicians sight-read a piece of music. The results suggest that structural differences of the score are reflected in the eye-hand span in a way similar to skill level. Specifically, the piece with higher complexity was associated with lower anticipation in notes, longer fixation duration and a tendency for more regressive fixations. Anticipation in time, however, remained the same $(\sim 1 \mathrm{~s})$ independently of the score played but was correlated with playing tempo. We conclude that the eye-hand span is not only influenced by the experience of the musician, but also by the structure of the score to be played.
\end{abstract}

M. Wiesendanger $(\square)$

Institute of Physiology, University of Fribourg,

Rue du Musée 5, 1700 Fribourg, Switzerland

e-mail: mario.wiesendanger@gmail.com

P. Wurtz $\cdot$ R. M. Mueri

Perception and Eye Movement Laboratory,

Departments of Clinical Research and Neurology, Inselspital, University of Bern, 3010 Bern, Switzerland

e-mail: pascal.wurtz@dkf.unibe.ch
Keywords Violin playing - Sight reading .

Music reading $\cdot$ Eye-hand span $\cdot$ Eye $\cdot$ Movements

\section{Introduction}

Welford, a well-known expert and author of motor control, with an interest in higher skills of music execution, wrote: “... the score is read and notes are played not singly but in whole phrases, and while one phrase is being played the next one is being read. The size of the group dealt with such tasks appears to increase with practice" (Welford 1976). The present report is about how violin players read music notation and execute the movements. Violinists face the tasks of delicate fingering with the left hand and of asymmetric bowing with the right arm. The term sight-reading is commonly used for reading the score, but should also hold for executing the score, since reading and playing are closely interconnected. As early as 1943, Weaver discovered that trained pianists alternated saccades (horizontal and vertical), with fixations to catch chords from the upper and lower staves of a piano score (Weaver 1943).

In a recent review on eye movements in everyday life, Land (2006) provided a short and concise overview on music reading, emphasizing the throughput rate of the highly skill-dependent processor. While discussing sightreading music, Land and Furneaux (1997) also suggest that ".... processed information is held in a memory buffer for a period of about 1 second. This permits a match between discontinuous input from the eyes and continuous motor output, and in particular allows the eyes to be involved in more than one task." Furneaux and Land (1999) also discovered that professional piano players increased the anticipation for capturing the notes with high 
skill while anticipation in time was related to the tempo rather than to skill. Kinsler and Carpenter (1995) furthermore suggest that the degree of utilization of a hypothetical memory buffer (between perceptual and motor processes) may determine the amplitude and timing of eye movements.

Sight-reading has become an important issue in music education in order to test groups of pianists with varying skill levels, including also high-level professional pianists (e.g., Kinsler and Carpenter 1995; Sloboda et al. 1998). The goal is clearly to practice anticipatory silent reading of a group of notes, followed by music execution. The anticipatory nature of discrete eye fixations creates maneuvring room for the player, allowing for smooth motor performance and audition. In line with this presumption Truitt et al. (1997) found larger eye-hand spans in skilled compared with less-skilled pianists. This interval between fixations and fingering, the so-called eye-hand span, is an essential parameter in our study.

In practical music teaching (e.g., Eales 1992), sight-reading covers only the reading part, often without recording motor execution. To our knowledge, formal sight-reading of music has been done exclusively in piano players. Music execution was conventionally transformed by means of an MIDI interface (musical instrument digital interface) to obtain the timing of each tone of the piano sequence. In the present study, we have been successful, for the first time, in measuring sight-reading in skilled violinists, by synchronizing the timing of eye fixations, as well as discrete bow-reversal points of the violinist with exact measures of the tone sequence. Sight-reading an already familiar score is likely to be easier than sight-reading a similar non-familiar score. The highest level of playing, completely from memory, can often be heard from famous soloists.

\section{Method \\ Participants}

Seven violinists, five female and two male (mean age 41 years, SD 22, range 23-76 years) volunteered to participate in the study. All had been trained, including in orchestra and ensembles, four among them being professionals. Handedness was assessed by the Edinburgh Inventory (Oldfield 1971), which ranges from -100 for strong lefthandedness to +100 for strong right-handedness. All subjects were right handed (mean 97, SD 4.8) and had normal (or corrected-to-normal) vision. The investigation was carried out in accordance with the Declaration of Helsinki with all participants giving written informed consent prior to the experiment.

\section{Materials and procedure}

All candidates were asked to play at their own tempo excerpts of two sonatas, one of Corelli and one of Telemann. The Corelli part (Fig. 1a) consisted exclusively of 1/16th (semiquaver) notes; 153 notes and bow strokes altogether. The Telemann part (Fig. 1b) consisted of 122 notes in total, including $1 / 8$ th notes (quavers), triple notes and 1/16th notes (semiquavers) and was played with 98 bow strokes. Each note had to be played with a separate bow stroke in the Corelli part, and most also in the Telemann part, with the exception of the triple notes played with one bow stroke each. We estimated the Telemann score to be more complex than the Corelli score because of (1) its less regular notation pattern and (2) its higher complexity of intermittent position changes and fast triple sequences. The music score was fastened on a cardboard $(21 \times 29.7 \mathrm{~cm})$ and fixed to the eye-tracker helmet (see Fig. 2a) at a distance of $39 \mathrm{~cm}$ in front of the eyes. The resulting viewing angle was approximately $40^{\circ} \times 30^{\circ}$.

Eye movements were recorded by means of an infrared-video based head-mounted eye tracker (HED ${ }^{\mathrm{TM}}$,

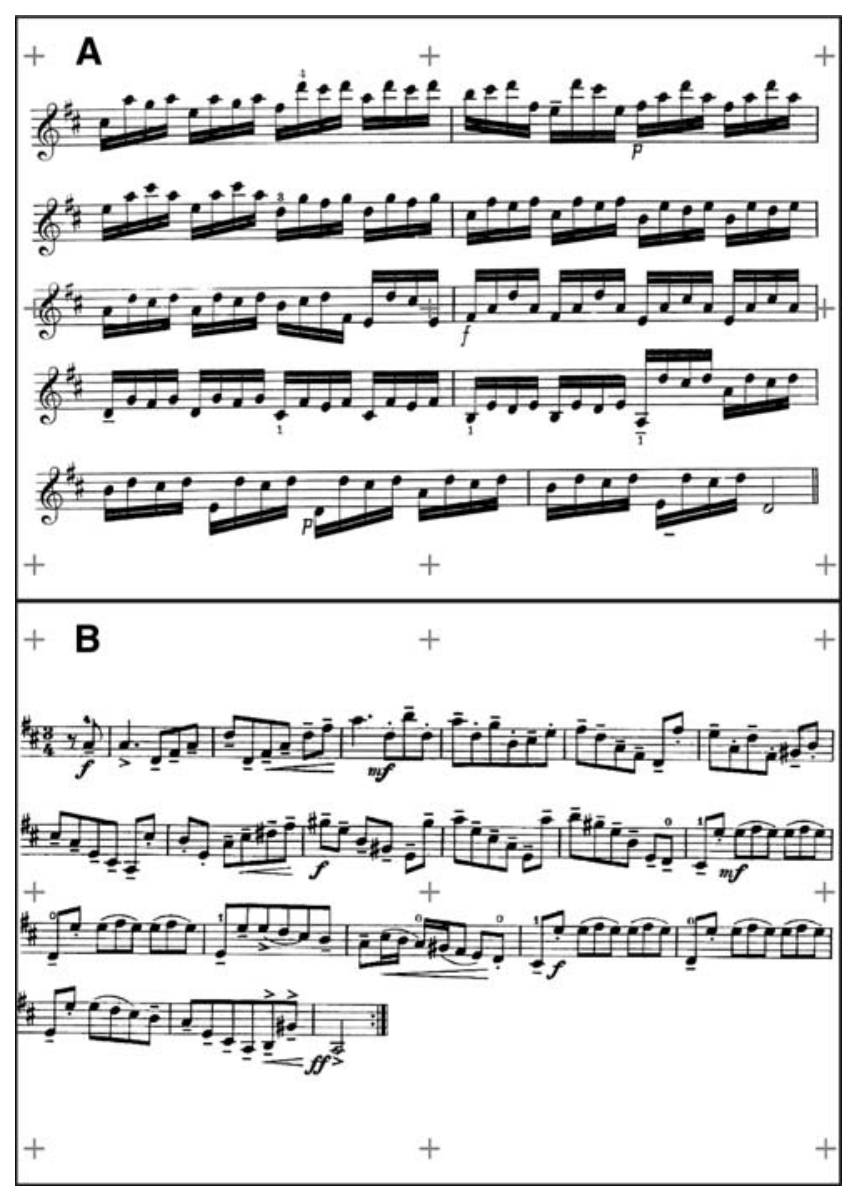

Fig. 1 a Telemann, b Corelli: the two scores to be played by the participants; the light gray crosses were used to calibrate the eye tracker 


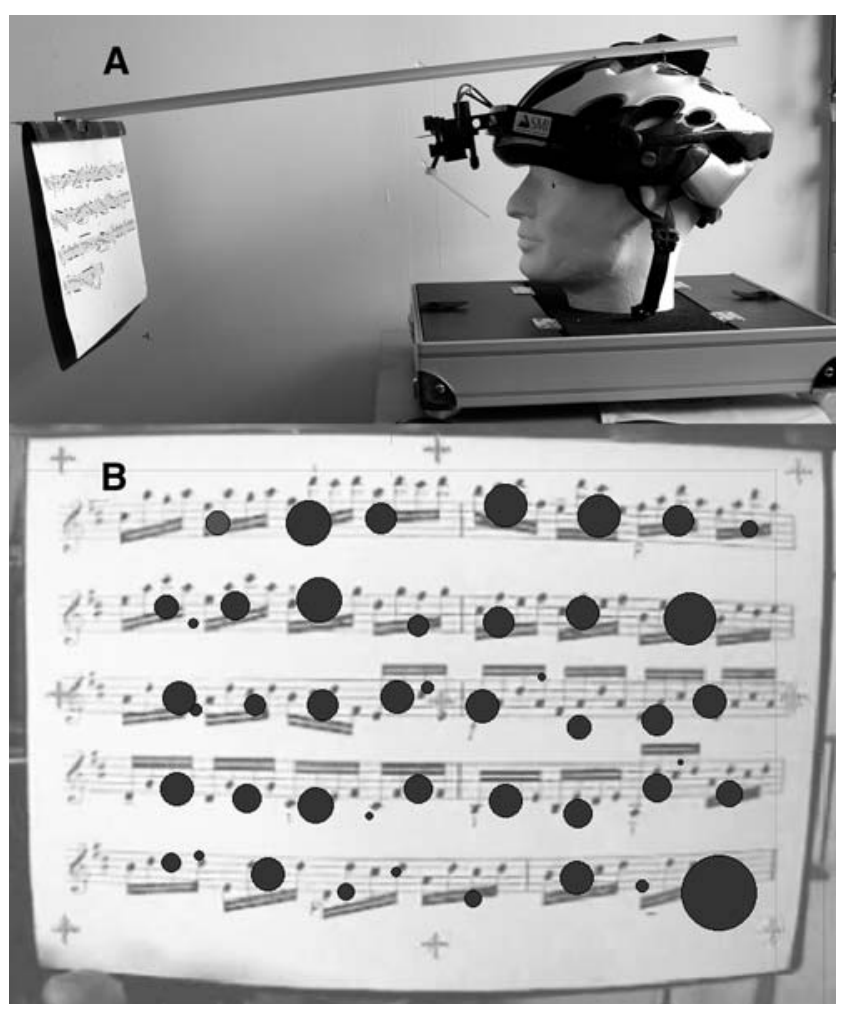

Fig. 2 a Head-mounted eyetracking device with the attached music score. b Illustration of the recorded eye fixations (black circles), drawn in proportion of the fixation duration

SensoMotoric Instruments GmbH, Teltow, Germany) with a temporal resolution of $50 \mathrm{~Hz}$ and tracking resolution $<0.1^{\circ}$. The system consists of one camera recording the scene with the score in front the subject and another camera recording the eye movements (Fig. 2a). Each experimental session was preceded by a 9-point calibration, resulting in a gazeposition accuracy of typically better than $1^{\circ}$. The eye tracker and an illustration of the resulting eye movement data are shown in Fig. 2.

The recording of bowing movements was achieved with the Vicon 460 motion system, consisting of four specialized cameras including infrared strobes that were reflected back from three markers fixed along the bow and three markers on the violin. The Vicon data station controlled the cameras and strobes and collected the incoming signals from all reflecting markers at $200 \mathrm{~Hz}$. These were finally transmitted and stored on a PC along with the Vicon data management system for offline analysis. In addition, a conventional video camera simultaneously recorded the active player, and the sound track. The Eyetracker and the Vicon system were synchronized by means of a bifurcated start signal (TTL). This allowed us to measure the eye- and bow movements with reference to the same time scale.

After the eye tracker had been fitted to the subject's head, the players had a few minutes to familiarize themselves with the setup of the score (fixed to the helmet) that followed the head movements. Finally, the eye tracker and the Vicon motion capture system were calibrated with the fixed music score. A go-signal synchronized the Vicon and Eyetracker and the subject started to play from the score. They were free to choose their tempo. All bow and eye movement data were stored for offline analysis. Figure 3 illustrates the bow movements of the Corelli and the Telemann scores which were obtained by the Vicon model of

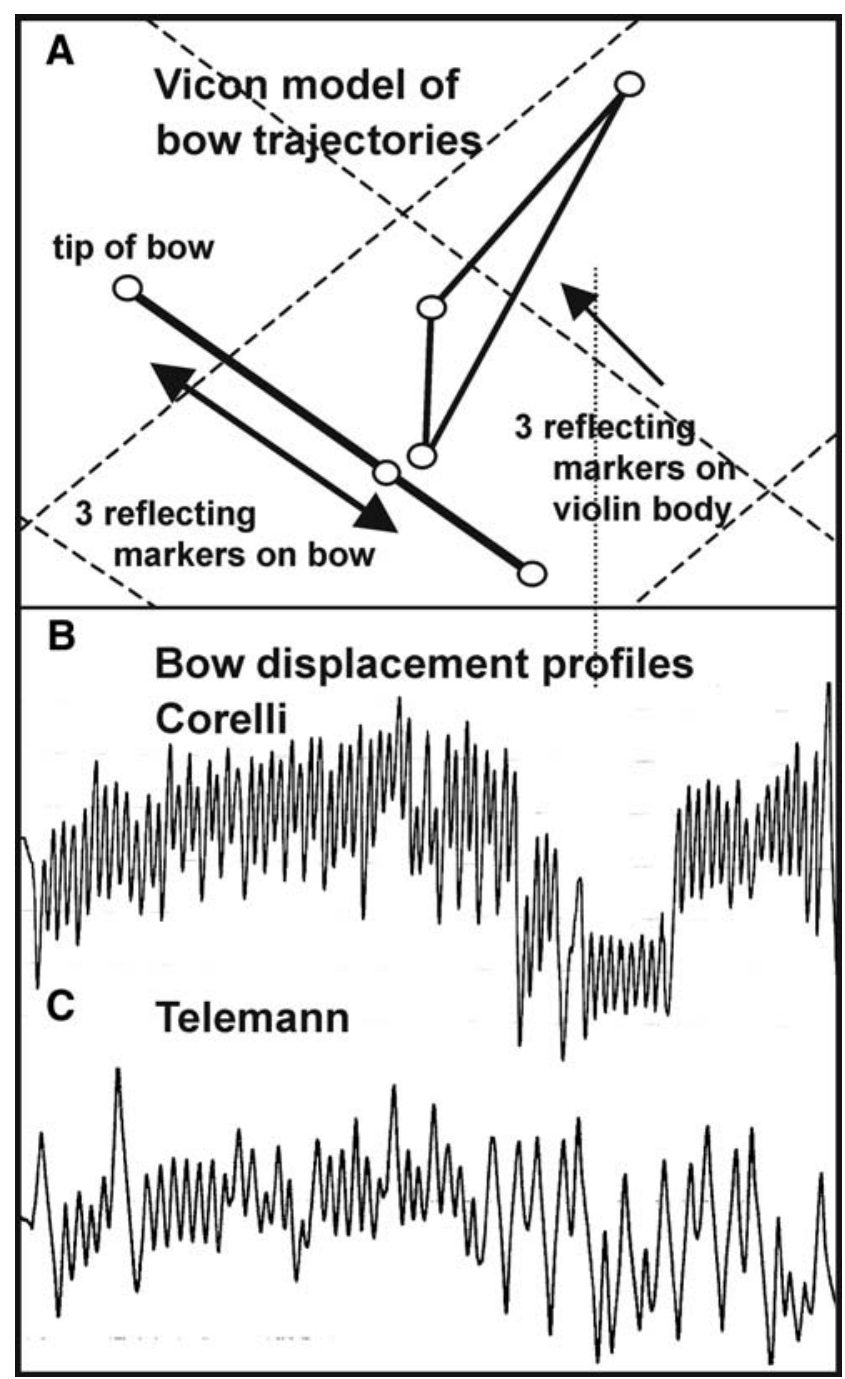

Fig. 3 a Vicon model for the bow movements. The markers placed on the bow (3) and on the violin (3) continuously receive and reflect the infrared strobes from the 4 Vicon cameras; thus, capturing the momentary position of each marker (resolution $200 \mathrm{~Hz}$ ). One of the marker (usually at the tip) was selected to obtain the displacement curves with the sharp, high resolution bow reversals. At each bow reversal a new tone (or a small group of tones in the Telemann score) is initiated, ending with the next tone. The space coordinates were embedded in the violin plate thus eliminating other body movements. b Bow displacement profiles of playing the Corelli score. c Same for Telemann score. The straight bow movements are interrupted with sharp bow reversals, each inducing the next tone (or small group of notes in the Telemann piece) 
the bow movements providing the discrete reversal points that generated the tone sequences.

\section{Data analysis}

The straight linear up- and down strokes of bowing were ideal for marking each reversal point indicating the onset of the next tone in the series of the 153 bow reversals for Corelli; the same procedure was also used for the 98 bow reversal points of the Telemann piece. This information allowed us to match each note (and a few small groups of notes) with the precise timing of the original playing.

In a second step, all fixations were projected onto a still image of the video scene and assigned to the nearest note on the sheet with reference to the same time scale. This format allowed us to compute the eye-hand span in two ways: (1) in terms of number of notes between the execution of a bow reversal and the simultaneous fixation; (2) in terms of time between fixation onset on a note and subsequently playing it (bow reversal as indicator of tone onset). Figure 4 illustrates the data analysis. In addition, general behavioral measures were registered for descriptive and correlational analysis. These included (1) the playing time (s) and (2) the mean fixation duration (mean period of relative stability between eye saccades), and finally the number of regressive saccades (i.e., eye movements from right to left, i.e., opposite to the reading direction).

All data are expressed as means \pm SD. If not stated differently, statistical mean comparisons were performed using $t$ tests. To correct for cumulative errors in multiple comparisons, Bonferroni correction was applied by adjusting the $P$ values accordingly. The significance level was set at $P<0.05$.

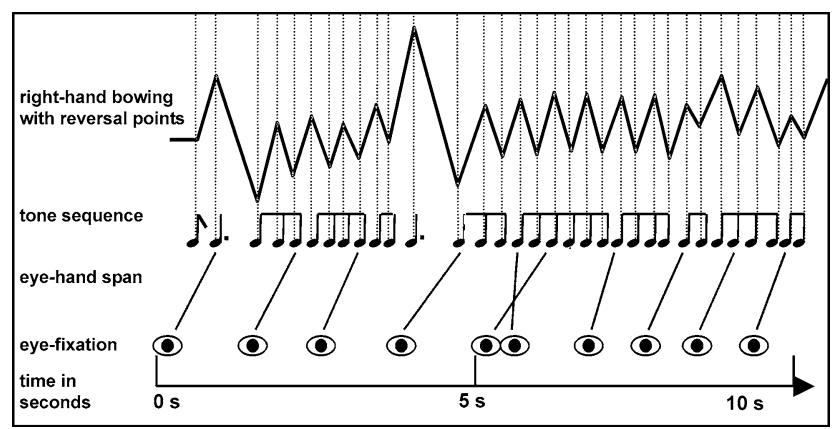

Fig. 4 Illustration of the recorded data and their analysis: the data are from one subject playing the first line of the Telemann score. The top line represents the straight bow strokes with the sharp bow-reversal points. All bow reversal-points (upper maxima and lower minima) each represent a tone onset which ends with the next bow reversal as illustrated by the vertical dotted lines. Eye fixations at the bottom are drawn at the time of their onset and connected to the visual capture of notes within each fixation. The difference (in seconds or in notes) between music performance and fixation represents the eye-hand-span. Note one fixation which is regressive (between seconds 5 and 6)

\section{Results}

Structural differences of the two scores

Musicians played, at their own pace, the two scores without a metronome. We questioned whether the timing differed as a result of the individual playing styles, the musical character of the two pieces, or of both. Therefore, we correlated the playing time of the Corelli and Telemann scores. It turned out that there was a strong correlation between the timing of the two scores: $r(7)=0.975, P<0.001$. This means that if the Corelli score was played fast, the Telemann score was also played fast thus indicating individual preferences in playing speed. The Corelli score consisted exclusively of semiquavers (1/16th notes), whereas the Telemann score was a mixture of more variable notes with changing durations. The total play time was significantly shorter for the Corelli (mean $28.5 \mathrm{~s}, \mathrm{SD}=7.3 \mathrm{~s}$ ) than for the Telemann score (mean $32.2 \mathrm{~s}, \mathrm{SD}=7.6 \mathrm{~s}), t(1.6)=-4.838$, $P=0.003$. Although the chosen Telemann score is more heterogeneous with variable tone durations, the Corelli Sonata was played with homogenous and fast semiquavers, favouring a higher tempo, as compared to the tempo of the more complex Telemann Sonata. In brief, the tempo difference is also a natural outcome of a simpler and faster notation in the selected extract of Corelli's sonata (but note that Corelli also composed a part of the same movement of the same sonata with a more complex and slow tempo).

About eye fixation, duration, and eye-hand span

Mean fixation duration was significantly shorter for the Corelli score, i.e., $349 \mathrm{~ms}, \mathrm{SD}=116$, as compared to the Telemann score, $417 \mathrm{~ms}$, SD 173, $P=0.042, t(1,6)=$ -2.572 . In playing the Corelli score, there was a tendency for less regressive fixations (gazing back to the left of its preceding fixation (mean 1.00, SD 0.82), as compared to the Telemann score $($ mean $=1.71, \mathrm{SD}=1.13) ; t(1,6)=$ 1.050 , n.s.). Means and standard deviations of the eye-hand span for each subject are given in Table 1 The group mean is shown in Fig. 5. Paired samples $t$ tests, with Bonferroni adjusted $P$ values, revealed a higher anticipation in notes in the Corelli score, as compared to the Telemann score: $t(1$, $6)=4.491, P=0.008)$, while anticipation in time was not different: $t(1,6)=-2.276$, n.s.).

Finally, we tested whether the playing time influenced the eye-hand span. The time needed to play the score was positively correlated with anticipation in time $r(14)=$ $0.851, P<0.001$, but not with anticipation in notes $(r(14)=$ -0.217 , n.s.). Thus, violinists playing slowly had also more time for anticipation and made use of it. The number of anticipated notes; however, was independent of playing speed. 
Table 1 Visual anticipation in number of notes and in time (s) for each subject and piece to be played

\begin{tabular}{llllll}
\hline \multicolumn{2}{l}{ Anticipation in notes } & & & \multicolumn{2}{l}{ Anticipation in time } \\
\cline { 1 - 2 } Number & Corelli & Telemann & & Corelli & Telemann \\
\hline 1 & 4.86 & 2.65 & & 0.965 & 1.033 \\
2 & 6.55 & 4.02 & & 1.898 & 2.172 \\
3 & 9.03 & 4.11 & & 1.230 & 1.216 \\
4 & 5.18 & 2.74 & & 0.751 & 0.869 \\
5 & 6.56 & 3.92 & & 1.101 & 1.137 \\
6 & 6.15 & 3.68 & & 1.341 & 1.404 \\
7 & 3.51 & 3.57 & & 0.809 & 1.270 \\
\hline
\end{tabular}

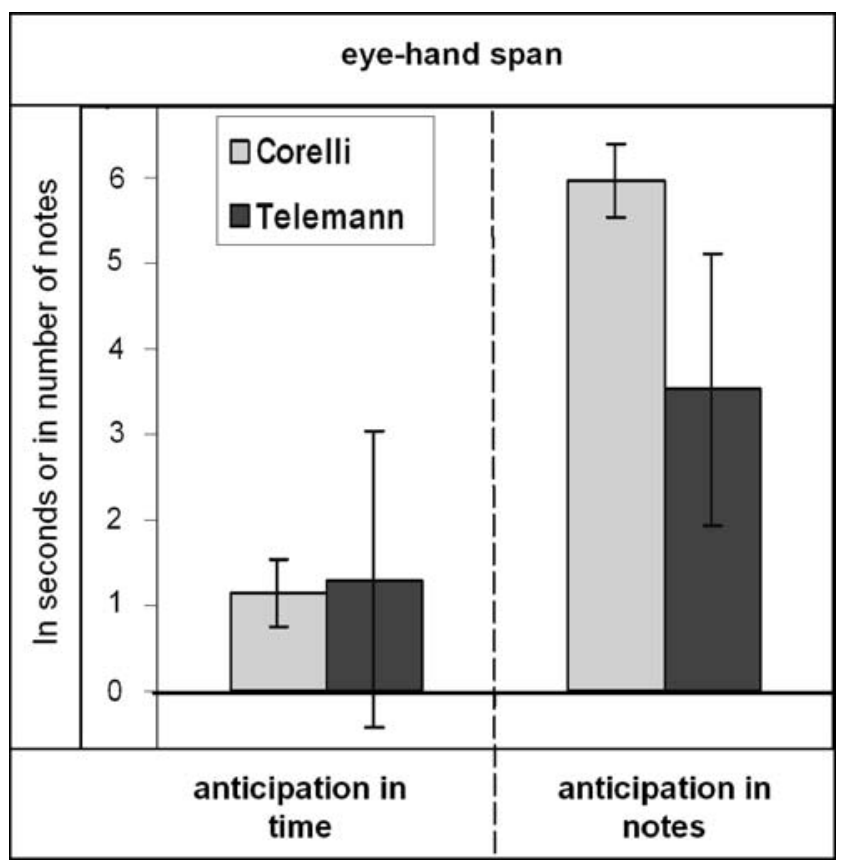

Fig. 5 During sight-reading, the eyes make progressive fixations (in this study of about $1 \mathrm{~s}$ ) during which one or more notes are read in anticipation, whereas the melody is unfolding continuously. The number of notes the eye is ahead of music execution is the eye-hand span

\section{Discussion}

Sight-reading is an essential cognitive visual-to-motor mechanism, rendering the music score. The visual-tomotor-to-hearing transformation is a complex neural and cognitive process, including anticipation, short-time memory, motor control and auditory feedback control. Score reading thus requires sequential and parallel processing of a vast amount of information in a very short time. Advanced musicians may also progressively memorize an entire score, but in music ensembles, particularly in an orchestra, the scores are usually present. In addition the players must watch the gestures of the conductor!
Earlier studies (i.e., Truitt et al. 1997) compared effects of performing skills and eye movement parameters. The interest of the present study was oriented towards effects of score complexities to oculomotor parameters and eye-handspans in a group of experienced violinists. Higher score complexity resulted in an oculomotor pattern with longer fixations and a tendency for more regressive fixations. This finding is consistent with similar reports in text reading (Rayner et al. 2006). We now extend it to formal score reading of violinists. Furthermore, this finding suggests that musical complexity already acts at a relatively early perceptual level before music execution. The observation that the playing time (i.e., tempo) depended on both the individual musician and the piece to be played, suggests that despite individual differences in preferred pace, playing time was adjusted to the musical content.

The main finding of our study was that anticipation in notes was lower for the more complex Telemann piece. At the same time the anticipation in time remained close to one second, independently of the score, but correlated with playing duration. It seems that a time window of $\sim 1 \mathrm{~s}$ is optimal for transforming visual information into motor performance. The higher processing demands of the Telemann piece were apparently compensated not by planning more but by reducing the anticipation in notes. In so doing fewer notes are packed into the hypothetical buffer (of about one second) and the subject has more time available for processing each note. This processing must contain both perceptual and motor processes. However; based on our data, we cannot discern the relative proportions.

The present data show that differences in the patterns of the two music scores are reflected in the musical execution of the players: the different rhythmic and melodic properties of the two pieces affected the way the musicians read and played the notes. Anticipation is not only influenced by the experience of the musician, but also by the interpretation of the score and its musical feeling. Finally, one should remark that the two pieces of music were short. The first movement of the Corelli Sonata, with exclusive groups of semi-quavers, was obviously less complex than the Telemann movement. But in the complete movement the Corelli sonata also changes from a fast to a slow, soft lyrical melody that was not played!

Sudnow (2001) remarks in his book “... as my hands began to form constellations, the scope of my looking correspondingly grasped the chord as a whole, seeing not its notes-for noteness, but for its configuration against the broader visual field of the terrain." In other words, one reads musical scores as chords or 'gestalt', not as single notes. To fixate repetitively a complex two-staved piano score (as for example Schumann's most difficult Toccata no. 7) is a particularly difficult task for sight-reading and playing; it requires an enormous experience, memory and talent. 
Violin playing includes skilful fingering with the left hand and bowing with the right arm. These completely asymmetric movements are difficult to master in a precise and smooth bimanual coordination. On a broader level, one has to learn and master the cognitive skills, including sightreading, and also substantial and precise motor skills in music execution. It is remarkable how the discrete intervals of short fixations with piece-meal memorization of note packages finally lead to a smooth flow of music, its performance and perception. As mentioned by Land and Furneaux (1997), “...the eyes are not only passively recording, but are also 'inquisitive' sensors." Motor control in playing music demands a high level of motor skill at its best! Violinists, as well as other musicians, are faced with a great amount of precision in fingering and bowing, position changes, string changes, tempo and intensity changes, all packed with emotional ingredients.

Acknowledgments We thank the violin players for their engagement and interest in this project which was done at the Institute of Physiology at the University of Fribourg, Switzerland. The authors would also like to thank Professor H. P. Clamann for comments and English proofreading on an earlier version of this manuscript. M. W. is particularly grateful for the fine collaboration with the Neurology Department, University of Berne, providing the experience with the eye tracker instrument.

\section{References}

Eales A (1992) The fundamentals of violin playing and teaching. In: Stowell R (ed) The cambridge companion to the violin. Cambridge University Press, Cambridge, pp 92-121

Furneaux S, Land MF (1999) The effects of skill on the eye-hand span during musical sight-reading. Proc Biol Sci 266:2435-2440

Kinsler V, Carpenter RHS (1995) Saccadic eye movements while reading music. Vis Res 35:1447-1458

Land MF (2006) Eye movements and the control of actions in everyday life. Prog Retina Eye Res 25:296-324

Land MF, Furneaux S (1997) The knowledge base of the oculomotor system. Philos Trans Roy Soc Lond B Biol Sci 352:1231-1239

Oldfield RC (1971) The assessment and analysis of handedness: the Edinburgh inventory. Neuropsychologia 9:97-113

Rayner K, Chace KH, Slattery TJ, Ashby J (2006) Eye movements as reflections of comprehension processes in reading. Sci Stud Read 10:241-255

Sloboda JA, Parncutt R, Clarke EF, Raekallio M (1998) Determinants of finger choice in piano sight-reading. J Exp Psychol 24:185-203

Sudnow D (2001) Ways of the hand-a rewritten account. MIT Press, Cambridge Mass, pp 0-139

Truitt FE, Clifton C, Pollatsek A, Rayner K (1997) The perceptual span and the eye-hand span in sight reading music. Vis Cogn 4:143161

Weaver HE (1943) A study of visual processes in reading differently constructed musical selections. Psychol Monogr 55:1-30

Welford AT (1976) Skilled performance: perceptual and motor skills. Scott Foresman Comp Glenview Ill. pp 1-200 